\title{
Mathematical modeling to reconstruct elastic and geoelectrical parameters
}

\author{
Vladimir N. Troyan and Yurii V. Kiselev \\ Institute of Physics, St. Petersburg State University, St. Petersburg, Russia
}

\begin{abstract}
The monitoring of the underground medium requires estimation of the accuracy of the methods used. Numerical simulation of the solution of 2D inverse problem on the reconstruction of seismic and electrical parameters of local (comparable in size with the wavelength) inhomogeneities by the diffraction tomography method based upon the first order Born approximation is considered. The direct problems for the Lame and Maxwell equations are solved by the finite difference method that allows us to take correctly into account the diffraction phenomenon produced by the target inhomogeneities with simple and complex geometry. For reconstruction of the local inhomogeneities the algebraic methods and the optimizing procedures are used. The investigation includes a parametric representation of inhomogeneities by the simple and complex functions. The results of estimation of the accuracy of the reconstruction of elastic inhomogeneities and inhomogeneities of electrical conductivity by the diffraction tomography method are represented.
\end{abstract}

Key words diffraction tomography - mathematical modeling - seismic tomography - electromagnetic tomography

\section{Introduction}

Diffraction tomography is an imaging technique that makes use of a large volume of input data (recorded traces) to produce the image of underground medium parameters with high spatial resolution. In contrast to ray tomography (travel time tomography), for which the resolution is connected with the Fresnel zone and the large number of the source-receiver pairs is required, diffraction tomography (Devaney,

Mailing address: Dr. Vladimir N. Troyan, Universitetskaya nab. 7/9, St. Petersburg, 199034 Russia; e-mail: troyan@hq.pu.ru
1984; Devaney and Zhang, 1991; Zhou et al., 1993; Ryzhikov and Troyan, 1994; Alumbaugh and Morrison, 1995; Kiselev and Troyan, 1997) provides information on the medium parameters with subwavelength resolution.

In our study (a complete review of development of the diffraction tomography is introduced in (Devaney and Zhang, 1991)) most attention is given to an estimation of the accuracy of multiparametric reconstruction of elastic parameters and reconstruction of an electrical conductivity with the use of sounding by elastic wave and electromagnetic wave correspondingly. As a tool for our investigation, we use numerical simulation in $2 \mathrm{D}$ space domain. The direct problem is solved by the finite difference method that allows us correctly to take into account the diffraction phenomenon produced by the target inhomogeneities. The linearized inverse problem is solved by the diffraction tomography method with the use of the first order Born approximation (Keller, 1969). 
The applicability of the first order Born approximation in diffraction tomography is shown (Slaney et al., 1984) by numerical simulation conducted on a single cylinder using analytical expressions for the exact scattering field.

Beylkin and Burridge (1990) describe the multiparametric inversion in the time domain in the cases of acoustic and elasticity on the basis of the generalized Radon transform. This approach requires a large number of the sourcereceiver pairs. We also implement multiparametric reconstruction in the time domain, but our numerical simulation is realized with not more than three sources and three receivers (nine source-receiver pairs). For the multiparametric reconstruction in the elastic case (reconstruction of the Lame parameters, mass density and as a corollary - shear and longitudinal velocities) the optimizing procedures are used. The multiparametric reconstruction makes use of amplitude information connected to the scattering characteristic (Wu and Aki, 1985; Beylkin and Burridge, 1990) of the elementary disturbances of the reconstructed parameters. Under tomography experiment, these scattering characteristics should be taken into account by the use of the relevant observation schemes. Scattering by the elementary disturbances of the parameters can be described with the use of tomography functionals (Ryzhikov and Troyan, 1994; Troyan and Ryzhikov, 1994), which in a form of the ray series are represented for elastic and electromagnetic cases.

\section{Basic equations and algorithms for elastic case}

The numerical simulation to reconstruct the parameters of local inhomogeneities with a smooth change of the elastic parameters $\lambda, \mu$ and mass density $\rho$ is carried out for two-dimensional model of the elastic medium, containing the free surface and plane-parallel welded interfaces.

The source $\hat{f} \equiv \hat{\boldsymbol{f}}(\boldsymbol{x}, t)$, located in the point $\left(x=x_{s}, z=z_{s}\right)$ of the Cartesian system of coordinates $\left(x, y, z ; \boldsymbol{e}_{1}, \boldsymbol{e}_{2}, \boldsymbol{e}_{3}\right)$, produces the wave field $\boldsymbol{u} \equiv \boldsymbol{u}(x, z, t) \equiv \boldsymbol{u}(\boldsymbol{x}, t)$ which satisfies the equation

$$
\begin{aligned}
& \rho \frac{\partial^{2} \boldsymbol{u}}{\partial t^{2}}=\hat{\boldsymbol{f}}+(\lambda+\mu) \nabla \nabla \cdot \boldsymbol{u}+\mu \Delta \boldsymbol{u}+ \\
& +\nabla \lambda \nabla \cdot \boldsymbol{u}+\nabla \mu \times \boldsymbol{u}+2(\nabla \mu \cdot \nabla) \boldsymbol{u} .
\end{aligned}
$$

Boundary condition at the free surface $(z=0)$ is

$$
\left.\boldsymbol{t}_{z}\right|_{z=0}=0
$$

and at welded interfaces $\left(z=z_{i}\right)$ we assume that

$\left.\boldsymbol{u}\right|_{z=z_{i}-0}=\left.\boldsymbol{u}\right|_{z=z_{i}+0},\left.\quad \boldsymbol{t}_{z}\right|_{z=z_{i}-0}=\left.\boldsymbol{t}_{z}\right|_{z=z_{i}+0}$.

The field $\boldsymbol{u}$ will be produced by the sources $\hat{\boldsymbol{f}}, \hat{\boldsymbol{f}}_{1}, \hat{\boldsymbol{f}}_{3}$

$$
\begin{gathered}
\hat{\boldsymbol{f}} \equiv \hat{\boldsymbol{f}}\left(x_{s}, z_{s}, t\right)=\delta\left(x-x_{s}\right) \delta\left(z-z_{s}\right) f(t) \boldsymbol{e}_{3} \\
\hat{\boldsymbol{f}}_{1} \equiv \hat{\boldsymbol{f}}_{1}\left(x_{r}, z_{r}, t\right)=\delta\left(x-x_{r}\right) \delta\left(z-z_{r}\right) \delta(t) \boldsymbol{e}_{1} \\
\hat{\boldsymbol{f}}_{3} \equiv \hat{\boldsymbol{f}}_{3}\left(x_{r}, z_{r}, t\right)=\delta\left(x-x_{r}\right) \delta\left(z-z_{r}\right) \delta(t) \boldsymbol{e}_{3}
\end{gathered}
$$

with the time dependences $f(t)$ and $\delta(t)$, which are located at the source point $\boldsymbol{x}=\left(x_{S}, z_{s}\right)$ and at the observation point $\boldsymbol{x}=\left(x_{r}, z_{r}\right)$. The velocities of longitudinal $\left(v_{p}\right)$ and shear $\left(v_{s}\right)$ waves, expressed through the quantities $\lambda, \mu, \rho$, read as

$$
v_{p}=\sqrt{\frac{\lambda+2 \mu}{\rho}}, \quad v_{s}=\sqrt{\frac{\mu}{\rho}} .
$$

We introduce the differences $\delta \lambda=\lambda-\lambda_{\mathrm{rf}}, \delta \mu=$ $\mu-\mu_{\mathrm{rf}}$ and $\delta \rho=\rho-\rho_{\mathrm{rf}}$ of the values $\lambda, \mu, \rho$, for the unknown medium, which are connected with 
the wave field $\boldsymbol{u}(\boldsymbol{x}, t)$

$$
\begin{gathered}
L \boldsymbol{u}=-\hat{f}, \quad L \boldsymbol{u} \equiv(\lambda+\mu) \nabla \nabla \cdot \boldsymbol{u}+\mu \Delta \boldsymbol{u}+ \\
: \nabla \lambda \nabla \cdot \boldsymbol{u}+\nabla \mu \times \boldsymbol{u}+2(\nabla \mu \cdot \nabla) \boldsymbol{u}-\rho \frac{\partial^{2} u}{\partial t^{2}}
\end{gathered}
$$

and the values $\lambda_{\mathrm{rf}}(\boldsymbol{x}), \mu_{\mathrm{rf}}(\boldsymbol{x}), \rho_{\mathrm{rf}}(\boldsymbol{x})$ for the known, reference (rf) medium for which the wave field is $\boldsymbol{u}_{\mathrm{rf}}(\boldsymbol{x}, t)$

$$
\begin{gathered}
L_{\mathrm{rf}} \boldsymbol{u}_{\mathrm{rf}}=-\hat{\boldsymbol{f}}, \\
L_{\mathrm{rf}} \boldsymbol{u}_{\mathrm{rf}} \equiv\left(\lambda_{\mathrm{rf}}+\mu_{\mathrm{rf}}\right) \nabla \nabla \cdot \boldsymbol{u}_{\mathrm{rf}}+\mu_{\mathrm{rf}} \Delta \boldsymbol{u}_{\mathrm{rf}}+ \\
+\nabla \lambda_{\mathrm{rf}} \nabla \cdot \boldsymbol{u}_{\mathrm{rf}}+\nabla \mu_{\mathrm{rf}} \times \boldsymbol{u}_{\mathrm{rf}}+2\left(\nabla \mu_{\mathrm{rf}} \cdot \nabla\right) \boldsymbol{u}_{\mathrm{rf}}-\rho_{\mathrm{rf}} \frac{\partial^{2} \boldsymbol{u}_{\mathrm{rf}}}{\partial t^{2}} .
\end{gathered}
$$

Assuming $\delta \lambda, \delta \mu$, and $\delta \rho$ are small we can write

$$
L_{\mathrm{rf}} \delta \boldsymbol{u} \approx-\delta L \boldsymbol{u}_{\mathrm{rf}}
$$

where $\delta \boldsymbol{u}=\boldsymbol{u}-\boldsymbol{u}_{\mathrm{rf}}$ is the difference field. The right hand side of (2.8)

$$
\begin{aligned}
\delta L \boldsymbol{u}_{\mathrm{rf}}=\nabla \times & \left(\delta \mu \nabla \times \boldsymbol{u}_{\mathrm{rf}}\right)+2 \sum_{j=1,3} \nabla \cdot\left(\delta \mu \nabla u_{\mathrm{rf} j}\right) \boldsymbol{e}_{j}+ \\
& +\nabla\left(\delta \lambda \nabla \cdot \boldsymbol{u}_{\mathrm{rf}}\right)-\delta \rho \frac{\partial^{2} \boldsymbol{u}_{\mathrm{rf}}}{\partial t^{2}}
\end{aligned}
$$

can be considered as a source of this field

We shall represent the components of the difference field $\delta u_{i}$ from (2.8) at the observation point of $\boldsymbol{x}=\boldsymbol{x}_{r}$, as following:

$$
\begin{gathered}
\delta u_{i}\left(\boldsymbol{x}_{s}, \boldsymbol{x}_{r}, t\right)= \\
=\int_{s} \int_{0}^{\infty} \tilde{\boldsymbol{u}}_{i}\left(\boldsymbol{x}, \boldsymbol{x}_{r}, t-\tau\right) \cdot \delta L \boldsymbol{u}\left(\boldsymbol{x}, \boldsymbol{x}_{r}, \tau\right) d \tau d \boldsymbol{x}
\end{gathered}
$$

where $S$ is the region of reconstruction; $\boldsymbol{u} \equiv \boldsymbol{u}\left(\boldsymbol{x}, \boldsymbol{x}_{s}, t\right)$ and $\tilde{\boldsymbol{u}_{1}} \equiv \tilde{\boldsymbol{u}_{1}}\left(\boldsymbol{x}, \boldsymbol{x}_{r}, t\right), \tilde{\boldsymbol{u}_{3}} \equiv \tilde{\boldsymbol{u}_{3}}\left(\boldsymbol{x}, \boldsymbol{x}_{r}, t\right)$ are the solutions of the eqs. (with sources from (2.4))

$$
L_{\mathrm{rf}} \boldsymbol{u}=-\hat{\boldsymbol{f}}
$$

and

$$
L_{\mathrm{rf}} \tilde{\boldsymbol{u}}_{i}=-\hat{\boldsymbol{f}}_{i} \quad(i=1,3)
$$

respectively.

After introducing the tomography functionals (Troyan and Ryzhikov, 1994)

$$
\begin{aligned}
& p_{i}^{\rho}\left(\boldsymbol{x}, \boldsymbol{x}_{r}, \boldsymbol{x}_{s}, t\right)= \\
& =-\int_{0}^{\infty} \tilde{\boldsymbol{u}}_{i}\left(\boldsymbol{x}, \boldsymbol{x}_{r}, t-\tau\right) \cdot \frac{\partial^{2}}{\partial \tau^{2}} \boldsymbol{u}\left(\boldsymbol{x}, \boldsymbol{x}_{s}, \tau\right) d \tau \\
& p_{i}^{\lambda}\left(\boldsymbol{x}, \boldsymbol{x}_{s}, \boldsymbol{x}_{r}, t\right)= \\
& =-\int_{0}^{\infty} \nabla \cdot \tilde{\boldsymbol{u}}_{i}\left(\boldsymbol{x}, \boldsymbol{x}_{r}, t-\tau\right) \nabla \cdot \boldsymbol{u}\left(\boldsymbol{x}, \boldsymbol{x}_{s}, t-\tau\right) d \tau \\
& p_{i}^{\mu}\left(\boldsymbol{x}, \boldsymbol{x}_{s}, \boldsymbol{x}_{r}, t\right)= \\
& =+\int_{0}^{\infty}\left[\nabla \times \tilde{\boldsymbol{u}}_{i}\left(\boldsymbol{x}, \boldsymbol{x}_{r}, t-\tau\right) \cdot \nabla \times \boldsymbol{u}\left(\boldsymbol{x}, \boldsymbol{x}_{s}, \tau\right)-\right. \\
& \left.-2 \sum_{j=1,3} \nabla \tilde{u}_{i j}\left(\boldsymbol{x}, \boldsymbol{x}_{r}, t-\tau\right) \cdot \nabla u_{j}\left(\boldsymbol{x}, \boldsymbol{x}_{s}, \tau\right)\right] d \tau
\end{aligned}
$$

the components of the difference field $\delta u_{i}(2.10)$ can be written down as

$$
\begin{gathered}
\delta u_{i}\left(\boldsymbol{x}_{s}, \boldsymbol{x}_{r}, t\right)=\int_{S}\left[\delta \lambda(\boldsymbol{x}) p_{i}^{\lambda}\left(\boldsymbol{x}, \boldsymbol{x}_{s}, \boldsymbol{x}_{r}, t\right)+\right. \\
\left.+\delta \mu(\boldsymbol{x}) p_{i}^{\mu}\left(\boldsymbol{x}, \boldsymbol{x}_{s}, \boldsymbol{x}_{r}, t\right)+\delta \rho(\boldsymbol{x}) p_{i}^{\rho}\left(\boldsymbol{x}, \boldsymbol{x}_{s}, \boldsymbol{x}_{r}, t\right)\right] d \boldsymbol{x} .
\end{gathered}
$$

Using the linear relations between $\delta \lambda(x), \delta \rho(x)$ and $\delta \mu(\boldsymbol{x})$

$$
\begin{gathered}
\delta \lambda(\boldsymbol{x})=c_{\lambda} \delta \mu(\boldsymbol{x}), \delta \rho(\boldsymbol{x})=c_{\rho} \delta \mu(\boldsymbol{x}), \\
\left(c_{\lambda}=\text { const }, c_{\rho}=\text { const }\right)
\end{gathered}
$$


the eq. (2.14) can be rewritten as

$$
\begin{gathered}
\delta u_{i}\left(\boldsymbol{x}_{s}, \boldsymbol{x}_{r}, t\right) \approx \int_{S}\left[c_{\lambda} p_{i}^{\lambda}\left(\boldsymbol{x}, \boldsymbol{x}_{s}, \boldsymbol{x}_{r}, t\right)+\right. \\
\left.+p_{i}^{\mu}\left(\boldsymbol{x}, \boldsymbol{x}_{s}, \boldsymbol{x}_{r}, t\right)+c_{\rho} p_{i}^{\rho}\left(\boldsymbol{x}, \boldsymbol{x}_{s}, \boldsymbol{x}_{r}, t\right)\right] \delta \mu(\boldsymbol{x}) d \boldsymbol{x} .
\end{gathered}
$$

After the digitization of the eq. (2.16), the system of equations for determination of $\delta \mu$ (vector $\left.d_{\mu}\right)$, $c_{\lambda}$ and $c_{\rho}$ can be written as

$$
P\left(c_{\lambda}, c_{\rho}\right) \boldsymbol{d}_{\mu}=\boldsymbol{d}_{u}
$$

where $\boldsymbol{d}_{u}$ are the samples of the scattered field. The final version of this system after introducing regularizing terms reads as

$$
\begin{gathered}
{\left[P^{\prime}\left(c_{\lambda}, c_{\rho}\right) P\left(c_{\lambda}, c_{\rho}\right)+\alpha_{1}\left(B_{x}^{\prime} B_{x}+B_{z}^{\prime} B_{z}\right)+\right.} \\
\left.+\alpha_{2} C^{\prime} C+\alpha_{3} D^{\prime} D\right] \boldsymbol{d}_{\mu}=P^{\prime}\left(c_{\lambda}, c_{\rho}\right) \boldsymbol{d}_{u}
\end{gathered}
$$

where $\alpha_{1}, \alpha_{2}, \alpha_{3}$ are the regularizing coefficients; matrices $B_{x}$ and $B_{z}$ are the finite difference images of second partial derivatives with respect to $x$ and $z$ correspondingly; $C$ and $D$ are penalty matrices for non-zero values of $\delta \mu$ at boundary and near boundary points of the reconstructed region $S$.

We find $\delta \mu, c_{\lambda}$ and $c_{\rho}$ by using an iterative procedure. At the first step the system of linear eqs. (2.18) is solved with some initial values $c_{\lambda}^{(0)}$ and $c_{\rho}^{(0)}$. By minimizing the sum of squared differences of the left-hand side and the right-hand side of (2.17), we find $c_{\lambda}^{(1)}$ and $c_{\rho}^{(1)}$, which are the corrected values of $c_{\lambda}^{(0)}$ and $c_{\rho}^{(0)}$. At the second step, the values $c_{\lambda}^{(1)}$ and $c_{\rho}^{(1)}$ are used for solution of the system of linear eqs. (2.18). The convergence of this procedure is based on the distinctions of the scattering diagrams (Wu and Aki, 1985) created by the elementary disturbances of $\lambda, \mu, \rho$. Similar distinctions can be studied, for example, using formulas (2.21) given below.

\subsection{Ray representation of the tomography functionals}

The components of the wave field $\delta u_{i}\left(\boldsymbol{x}_{s}, \boldsymbol{x}_{r}, t\right)$ $(i=1,3)$ scattered by local inhomogeneity $(\delta \lambda, \delta \mu, \delta \rho)$ for incident wave field $\boldsymbol{u}\left(\boldsymbol{x}, \boldsymbol{x}_{s}, t\right)$ are given by relations (2.14), (2.13).

Using the ray method the fields $\boldsymbol{u}\left(\boldsymbol{x}, \boldsymbol{x}_{s}, t\right)$, $\tilde{\boldsymbol{u}}_{i}\left(\boldsymbol{x}, \boldsymbol{x}_{r}, t\right)(2.11)-(2.13)$ can be represented as following:

$$
\begin{gathered}
\tilde{\boldsymbol{u}}_{i}\left(\boldsymbol{x}, \boldsymbol{x}_{r}, t\right)=\sum_{j=0}^{\infty} \sum_{q=p, s} \tilde{\boldsymbol{A}}_{i q j}\left(\boldsymbol{x}, \boldsymbol{x}_{r}, t\right) \tilde{f}_{j}\left(t-\tilde{\tau}_{q}\right) \\
\boldsymbol{u}\left(\boldsymbol{x}, \boldsymbol{x}_{s}, t\right)=\sum_{j=0}^{\infty} \sum_{q=p, s} \boldsymbol{A}_{q j}\left(\boldsymbol{x}, \boldsymbol{x}_{s}, t\right) f_{j}\left(t-\tau_{q}\right) \\
f_{j}(t)=f_{j+1}^{\prime}(t), \quad \tilde{f}_{j}(t)=\tilde{f}_{j+1}^{\prime}(t)
\end{gathered}
$$

where $\tilde{\tau}_{q} \equiv \tilde{\tau}_{q}\left(\boldsymbol{x}, \boldsymbol{x}_{r}\right)\left(\tau_{q} \equiv \tau_{q}\left(\boldsymbol{x}, \boldsymbol{x}_{S}\right)\right)$ is the time of propagation of a wave from point $\boldsymbol{x}_{r},\left(\boldsymbol{x}_{S}\right)$ to a point $\boldsymbol{x}$. Substituting of the relations (2.19) in (2.13) we write down the quantities $p_{i}^{\rho}, p_{i}^{\lambda}, p_{i}^{\mu}$ in the form of the ray series

$$
\begin{gathered}
p_{i}^{\rho}=\sum_{j=0}^{\infty} \sum_{q=p, s} \sum_{q^{\prime}=p, s} p_{i q q^{\prime} j}^{\rho} \phi_{j}\left(t-\tilde{\tau}_{q}-\tau_{q^{\prime}}\right) \\
p_{i}^{\lambda}=\sum_{j=0}^{\infty} \sum_{q=p, s} \sum_{q^{\prime}=p, s} p_{i q q^{\prime} j}^{\lambda} \phi_{j}\left(t-\tilde{\tau}_{q}-\tau_{q^{\prime}}\right) \\
p_{i}^{\mu}=\sum_{j=0}^{\infty} \sum_{q=p, s} \sum_{q^{\prime}=p, s} p_{i q q^{\prime} j}^{\mu} \phi_{j}\left(t-\tilde{\tau}_{q}-\tau_{q^{\prime}}\right) \\
\phi_{j}(t)=\phi_{j+1}^{\prime}(t), \quad \phi_{0}(t)=\frac{d^{2}}{d t^{2}} \int_{0}^{\infty} \tilde{f}_{0}(t-\tau) f_{0}(\tau) d \tau
\end{gathered}
$$

thus the coefficients $p_{i q q^{\prime} j}^{\lambda}, p_{i q q^{\prime} j}^{\mu}, p_{i q q^{\prime} j}^{\rho}(j=0) \mathrm{read}$ as

$$
\begin{aligned}
& p_{i q q^{\prime} 0}^{\rho}=-\left(\tilde{\boldsymbol{A}}_{i q 0} \cdot \boldsymbol{A}_{q^{\prime} 0}\right) \\
& p_{i q q^{\prime} 0}^{\lambda}=-\left(\tilde{\boldsymbol{A}}_{i q 0} \cdot \nabla \tilde{\tau}_{q}\right)\left(\boldsymbol{A}_{q^{\prime} 0} \cdot \nabla \tau_{q^{\prime}}\right) \\
& p_{i q q^{\prime} 0}^{\mu}=\left[\nabla \tilde{\tau}_{q} \times \tilde{\boldsymbol{A}}_{i q 0}\right] \cdot\left[\nabla \tau_{q^{\prime}} \times \boldsymbol{A}_{q^{\prime} 0}\right]- \\
& \quad-2\left(\tilde{\boldsymbol{A}}_{i q 0} \cdot \boldsymbol{A}_{q^{\prime} 0}\right)\left(\nabla \tilde{\tau}_{q} \cdot \nabla \tau_{q^{\prime}}\right) .
\end{aligned}
$$




\section{Basic equations and algorithms for electromagnetic case}

Numerical simulation to reconstruct the local inhomogeneities of electrical conductivity $\sigma=\sigma(x)$, located in the uniform space (electrical conductivity $\sigma=$ const, electrical permittivity $\varepsilon^{\prime}=\varepsilon \varepsilon_{0}=$ const, magnetic permittivity $\mu^{\prime}=\mu \mu_{0}$ $=$ const $)$ is implemented for the $2 \mathrm{D}$ problem. Electrical $(\boldsymbol{E}=\boldsymbol{E}(\boldsymbol{x}, t))$ and magnetic $(\boldsymbol{H}=\boldsymbol{H}(\boldsymbol{x}, t))$ fields excited by a current density $\boldsymbol{j}_{e x}=\boldsymbol{j}_{e x}(\boldsymbol{x}, t)$ satisfy to the Maxwell equations

$$
\begin{aligned}
& \nabla \times \boldsymbol{E}=-\frac{\partial \boldsymbol{B}}{\partial t}, \nabla \cdot \boldsymbol{B}=0, \boldsymbol{D}=\varepsilon^{\prime} \boldsymbol{E}, \boldsymbol{j}=\sigma \boldsymbol{E} \\
& \nabla \times \boldsymbol{H}=\boldsymbol{j}+\boldsymbol{j}_{e x}+\frac{\partial \boldsymbol{D}}{\partial t}, \nabla \cdot \boldsymbol{D}=\rho, \boldsymbol{B}=\mu^{\prime} \boldsymbol{H} .
\end{aligned}
$$

Electrical field $\boldsymbol{E}=\boldsymbol{E}(\boldsymbol{x}, t)$ in the medium, containing the local inhomogeneity $(\sigma=\sigma(x)$, $\left.\varepsilon^{\prime}=\varepsilon^{\prime}(\boldsymbol{x}), \mu^{\prime}=\mu^{\prime}(\boldsymbol{x})\right)\left({ }^{1}\right)$ is given by a solution of the equation

$$
\begin{gathered}
L \boldsymbol{E}=-\frac{\partial}{\partial t} \boldsymbol{j}_{e x} \\
L \boldsymbol{E}=\frac{1}{\mu^{\prime}} \nabla \times \nabla \times \boldsymbol{E}+\varepsilon^{\prime} \frac{\partial^{2} \boldsymbol{E}}{\partial t^{2}}+\sigma \frac{\partial \boldsymbol{E}}{\partial t}- \\
-\frac{1}{\mu^{\prime 2}}\left[\nabla \mu^{\prime} \times \nabla \times \boldsymbol{E}\right] .
\end{gathered}
$$

The reference medium (rf) is supposed to be known $\left(\sigma_{\mathrm{rf}}, \varepsilon_{\mathrm{rf}}, \mu_{\mathrm{rf}}\right)$ and electrical field $\boldsymbol{E}_{\mathrm{rf}}$ satisfies to the equation

$$
\begin{gathered}
L_{\mathrm{rf}} \boldsymbol{E}_{\mathrm{rf}}=-\frac{\partial}{\partial t} \boldsymbol{j}_{e x} \\
L_{\mathrm{rf}} \boldsymbol{E}_{\mathrm{rf}}=\frac{1}{\mu_{\mathrm{rf}}} \nabla \times \nabla \times \boldsymbol{E}_{\mathrm{rf}}+\varepsilon_{\mathrm{rf}} \frac{\partial^{2} \boldsymbol{E}_{r f}}{\partial t^{2}}+ \\
+\sigma_{\mathrm{rf}} \frac{\partial \boldsymbol{E}_{\mathrm{rf}}}{\partial t}-\frac{1}{\mu_{\mathrm{rf}}^{2}}\left[\nabla \mu_{\mathrm{rf}} \times \nabla \times \boldsymbol{E}_{\mathrm{rf}}\right] .
\end{gathered}
$$

As in the case of scattering by elastic inhomogeneities discussed earlier, we assume that magnitudes of the values $\delta \sigma=\sigma-\sigma_{\mathrm{rf}}, \delta \varepsilon=\varepsilon^{\prime}-\varepsilon_{\mathrm{rf}}$ and $\delta \mu=\mu^{\prime}-\mu_{\mathrm{rf}}$ make it possible to write an approximate equality

$$
L_{\mathrm{rf}} \delta E \approx-\delta L E_{\mathrm{rf}}
$$

where $\delta \boldsymbol{E}=\boldsymbol{E}-\boldsymbol{E}_{\mathrm{rf}}$ is the difference field. Thus, the value

$$
\delta L \boldsymbol{E}_{\mathrm{rf}}=\delta \varepsilon \frac{\partial^{2} \boldsymbol{E}_{\mathrm{rf}}}{\partial t^{2}}+\delta \sigma \frac{\partial \boldsymbol{E}_{\mathrm{rf}}}{\partial t}-\nabla \times\left(\frac{\delta \mu}{\mu_{\mathrm{rf}}^{2}} \nabla \times \boldsymbol{E}_{\mathrm{rf}}\right)
$$

can be considered as a source of this field. The components of the difference field $\delta E_{i}$ is possible to write down as

$$
\begin{gathered}
\delta E_{i}\left(\boldsymbol{x}_{s}, \boldsymbol{x}_{r}, t\right)= \\
=\int_{S}^{\infty} \int_{0}^{\infty} \tilde{\boldsymbol{E}}_{i}\left(\boldsymbol{x}, \boldsymbol{x}_{r}, t-\tau\right) \cdot \delta L \boldsymbol{E}\left(\boldsymbol{x}, \boldsymbol{x}_{s}, \tau\right) d \tau d \boldsymbol{x}
\end{gathered}
$$

that coinciding to within notations with (2.10). Wave fields $\boldsymbol{E}\left(\boldsymbol{x}, \boldsymbol{x}_{s}, \tau\right)$ and $\tilde{\boldsymbol{E}}_{i}\left(\boldsymbol{x}, \boldsymbol{x}_{r}, t-\tau\right)$ satisfy, respectively, the following equations:

$$
L_{\mathrm{rf}} \boldsymbol{E}=-\hat{\boldsymbol{f}} \quad \text { and } \quad L_{\mathrm{rf}} \tilde{\boldsymbol{E}}_{i}=-\hat{\boldsymbol{f}}_{i} \quad(i=1,3)
$$

where in a two-dimensional case the sources from (2.4) are used.

Introducing the tomography functionals

$$
\begin{aligned}
& p_{i}^{\varepsilon}\left(\boldsymbol{x}, \boldsymbol{x}_{r}, \boldsymbol{x}_{s}, t\right)=\int_{0}^{\infty} \tilde{\boldsymbol{E}}_{i}\left(\boldsymbol{x}, \boldsymbol{x}_{r}, t-\tau\right) \cdot \frac{\partial^{2}}{\partial \tau^{2}} \boldsymbol{E}\left(\boldsymbol{x}, \boldsymbol{x}_{s}, \tau\right) d \tau \\
& p_{i}^{\sigma}\left(\boldsymbol{x}, \boldsymbol{x}_{r}, \boldsymbol{x}_{s}, t\right)=\int_{0}^{\infty} \tilde{\boldsymbol{E}}_{i}\left(\boldsymbol{x}, \boldsymbol{x}_{r}, t-\tau\right) \cdot \frac{\partial}{\partial \tau} \boldsymbol{E}\left(\boldsymbol{x}, \boldsymbol{x}_{s}, \tau\right) d \tau \\
& p_{i}^{\mu}\left(\boldsymbol{x}, \boldsymbol{x}_{r}, \boldsymbol{x}_{s}, t\right)=-\int_{0}^{\infty} \nabla \times \tilde{\boldsymbol{E}}_{i}\left(\boldsymbol{x}, \boldsymbol{x}_{r}, t-\tau\right) \cdot \nabla \times \boldsymbol{E}\left(\boldsymbol{x}, \boldsymbol{x}_{s}, \tau\right) d \tau
\end{aligned}
$$

(') The main relations considered below will be written down in general case, i.e. we will assume an existance of anomalies of electrical permittivity and magnetic permittivity. 
the components of the difference field can be written as

$$
\begin{gathered}
\delta E_{i}\left(\boldsymbol{x}_{s}, \boldsymbol{x}_{r}, t\right) \equiv \delta E_{i}= \\
=\int_{S}\left(\delta \varepsilon p_{i}^{\varepsilon}+\delta \sigma p_{i}^{\sigma}+\delta \mu p_{i}^{\mu} / \mu_{\mathrm{rf}}^{2}\right) d \boldsymbol{x} .
\end{gathered}
$$

We will consider the numerical experiments on reconstruction of the anomalies of electrical conductivity $\sigma(\delta \varepsilon=0, \delta \mu=0)$. In this case, after the digitization, the integral eq. (3.9) can write as the system of linear equations

$$
P \boldsymbol{d}_{\sigma}=\boldsymbol{d}_{E}
$$

with respect to vector $\boldsymbol{d}_{\sigma}$, which is sought for the value $\delta \sigma(\boldsymbol{x})$, where $\boldsymbol{d}_{E}$ is the time samples of the components of the wave field scattered by inhomogeneity. The final version of these equations, after introducing the regularization terms, is coincident with the system of linear equations similar to (2.18).

\subsection{Ray representation of the tomography functionals}

In Section 2.1 the expressions for the tomography functionals (2.13) were written out in the case of the ray description of the wave fields $\boldsymbol{u}$ and $\tilde{\boldsymbol{u}}_{i}$, which are included into the integrands of the right-hand sides of (2.13). By assuming that the value of electrical conductivity for the reference medium is equal to zero, we can represent the wave fields $\boldsymbol{E}$ and $\tilde{\boldsymbol{E}}_{i}$ from (3.8) as

$$
\begin{gathered}
\tilde{\boldsymbol{E}}_{i}=\sum_{n=0}^{\infty} \tilde{\boldsymbol{A}}_{n i} \tilde{f}_{n}(t-\tilde{\boldsymbol{\tau}}), \quad \boldsymbol{E}=\sum_{n=0}^{\infty} \boldsymbol{A}_{n} f_{n}(t-\tau), \\
f_{n}(t)=f_{n+1}^{\prime}(t), \quad \tilde{f}_{n}(t)=\tilde{f}_{n+1}^{\prime}(t)
\end{gathered}
$$

and can write the tomography functionals (3.8) in a form of the ray series

$$
\begin{gathered}
p_{i}^{\varepsilon}=\sum_{n=0}^{\infty} p_{n i}^{\varepsilon} \phi_{n}(t-\tilde{\tau}-\tau) \\
p_{i}^{\sigma}=\sum_{n=0}^{\infty} p_{n i}^{\sigma} \phi_{n+1}(t-\tilde{\tau}-\tau) \\
p_{i}^{\mu}=\sum_{n=0}^{\infty} p_{n i}^{\mu} \phi_{n}(t-\tilde{\tau}-\tau) \\
\phi_{0}(t)=\frac{d^{2}}{d t^{2}} \int_{0}^{\infty} \tilde{f}_{0}(t-\tau) f_{0}(\tau) d \tau, \quad \phi_{n}(t)=\phi_{n+1}^{\prime}(t) .
\end{gathered}
$$

In a case of $n=0$ the values $p_{0 i}^{\sigma}, p_{0 i}^{\varepsilon}$ and $p_{0 i}^{\mu}$ are represented by the amplitude factors of the zero approximation $\tilde{\boldsymbol{A}}_{0 i}, \boldsymbol{A}$ and eikonals $\nabla \tilde{\boldsymbol{\tau}}, \nabla \tau$ (from (3.11))

$$
\begin{gathered}
p_{0 i}^{\varepsilon}=p_{0 i}^{\sigma}=\left(\tilde{\boldsymbol{A}}_{0 i} \cdot \boldsymbol{A}_{0}\right) \\
p_{0 i}^{\mu}=-\left(\left[\nabla \tilde{\boldsymbol{\tau}} \times \tilde{\boldsymbol{A}}_{0 i}\right] \cdot\left[\nabla \boldsymbol{\tau} \times \boldsymbol{A}_{0}\right]\right) .
\end{gathered}
$$

The values $p_{0 i}^{\sigma}, p_{0 i}^{\varepsilon}, p_{0 i}^{u}($ from (3.13)) describe the space characteristics of the scattered fields, which are produced by the elementary inhomogeneities $(\delta \sigma, \delta \varepsilon, \delta \mu)$ at the far-field region. The directivity diagrams in the cases of scattering by perturbation of electrical conductivity and electrical permittivity coincide. The wave field scattered by perturbation of electrical permittivity contains higher frequencies than the wave field scattered by perturbation of electrical conductivity.

It should be noted that formula (3.13) for the values $p_{0 i}^{\sigma}$ and $p_{0 i}^{\varepsilon}$ coincides with the formula for scattering the elastic wave field by the elementary perturbation of mass density $\left(^{2}\right)$.

$\left.{ }^{2}\right)$ Graphic representation of directivity diagrams for the elastic and electromagnetic cases can be found in Wu and Aki (1985) and Saintenoy and Tarantola (2001). 


\section{Numerical simulation}

\subsection{Elastic case}

As the result of numerical simulation, we obtain the values $\delta \lambda, \delta \mu$ and $\delta \rho$. With formula from (2.5) we get value of $\delta v_{p}$. The observation scheme and the location of inhomogeneity are represented in fig. 1 . The sources and observation points (9 pairs) are located at the free surface.

The results of reconstruction of $v_{p}$ are represented in figs. $2 a-d$ and $3 a-d$. Figure $2 a$ and fig. 3a show the models of two inhomogeneities with $20 \%$ contrast relatively to the reference medium. These inhomogeneities are located inside the layer with intermediate velocity. Figure $2 \mathrm{~b}, \mathrm{c}$ and fig. $3 \mathrm{~b}$ show the results of reconstruction obtained by the solution of the system of eqs. (2.18) with the different values of the regularizing coefficients. The relative error of the reconstruction in these cases is $20-25 \%$ (with the use of two components of the wave field). For the cases of more contrast inhomogeneities (20-40\%) the accuracy of reconstruction can be $\sim 50 \%$. Figure $2 \mathrm{~d}$ and fig. $3 \mathrm{c}, \mathrm{d}$ show the results of reconstruction using one (fig. $2 \mathrm{~d}$ and fig. $3 \mathrm{~d}$ ) or three (fig. 3c) parametric functions. In these cases, we solve the system of the eqs. (2.17) by minimization of the sum of squared differences between the left-hand side and the right handside of (2.17). The reconstruction with the use of just one simple parametric function is very stable.

From numerical simulation, we conclude that the realization of the diffraction tomography method offered in Ryzhikov and Troyan (1994), under appropriate observation scheme, allows satisfactory accuracy for velocity parameter reconstruction in the case of not very contrast inhomogeneities of size of $\sim \lambda_{p}$ at small number of source-receiver pairs.

\subsection{Electromagnetic case}

The model of inhomogeneity of electrical conductivity together with the results of reconstruction are represented in fig. $4 \mathrm{a}-\mathrm{c}$. The inhomogeneity, comparable in size with the wavelength of the sounding signal, is located inside the uniform space (reference medium) with parameters: $\varepsilon=10, \mu=1, \sigma=0$. The maximum

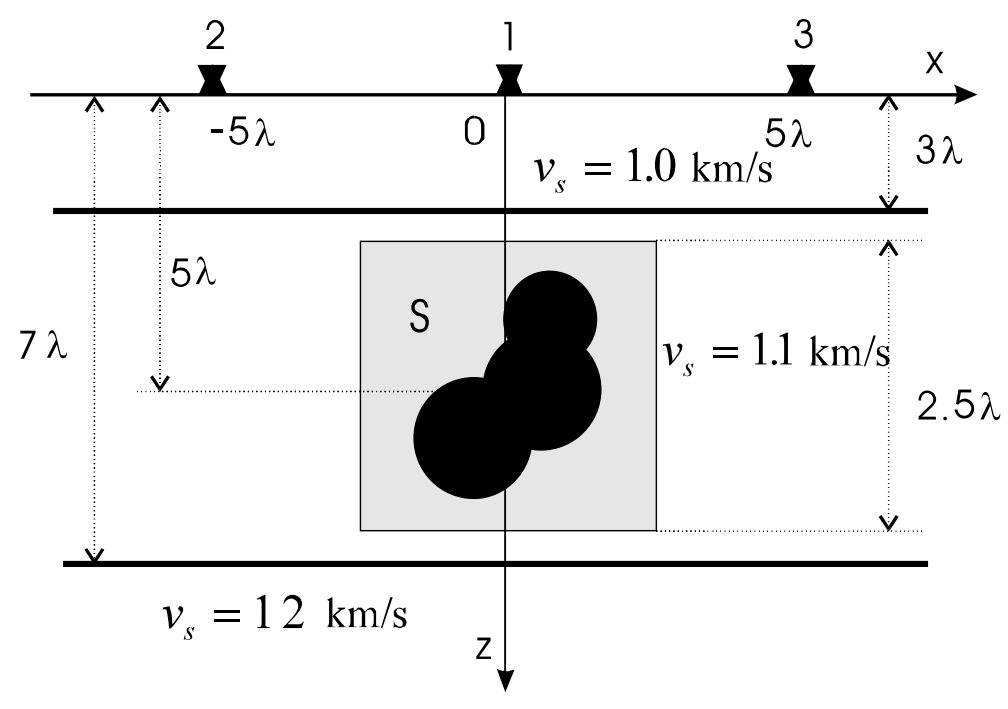

Fig. 1. Model of medium and observation scheme. $S$ is the region for reconstruction; location of inhomogeneity black color; $1,2,3$ are the source and the observation points locations; $\lambda \equiv \lambda_{s} ; v_{s} / v_{p}=1 / \sqrt{3}$. 


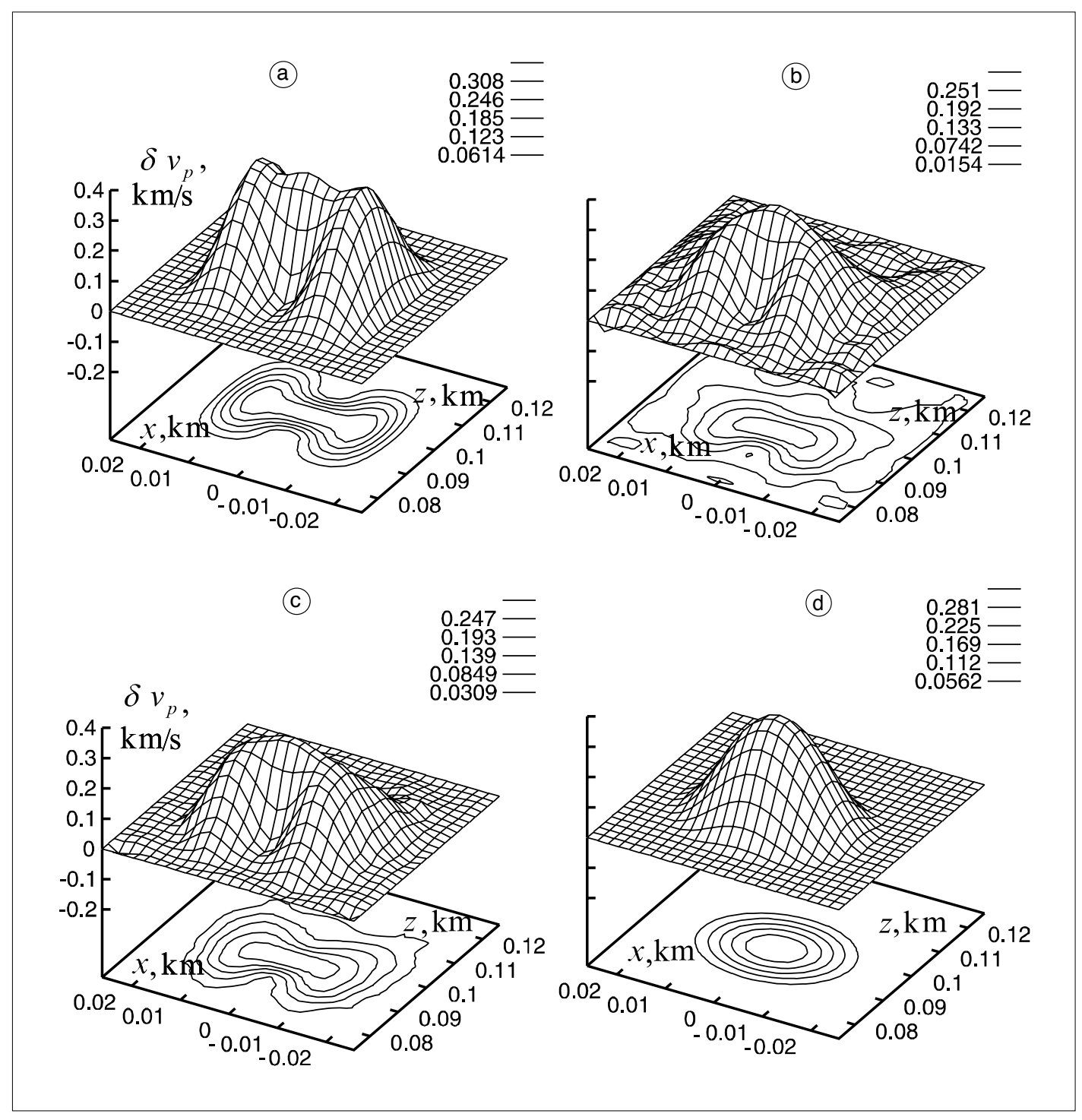

Fig. 2a-d. Reconstruction of $v_{p}$ for symmetric inhomogeneity. a) The model; b,d) results of reconstruction; b,c) solution of the system (2.18) with $\alpha_{3}=0$ and $\alpha_{3} \neq 0$ respectively; d) reconstruction in case of representation of reconstructed inhomogeneity by simple parametric function.

value of electrical conductivity is $10^{-3} \mathrm{~S} / \mathrm{m}$. An apparent frequency of the sounding signal is $5 \times 10^{6} \mathrm{~Hz}$. We use the observation scheme which is similar to the observation scheme represented in fig. 1. Distance between the observation line and the center of inhomogeneity is $10 \lambda(\lambda=20$ $\mathrm{m})$. The system of the linear eqs. (3.10) is solved under introducing the minimum magnitudes of 


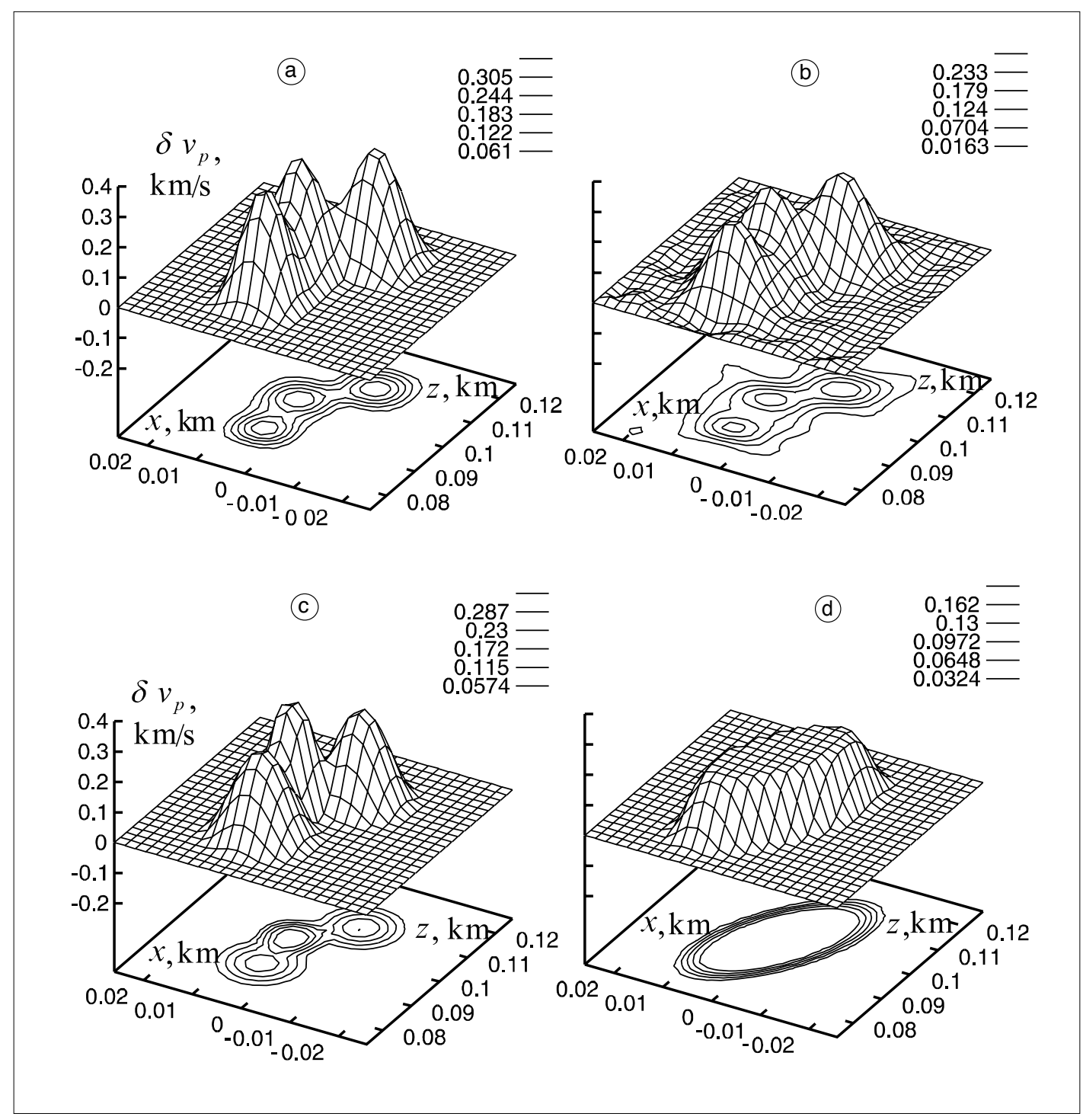

Fig. 3a-d. Reconstruction of $v_{p}$ for asymmetric inhomogeneity. a) The model; b,d) results of reconstruction; b) solution of the system of eq. (2.18) with $\alpha_{3}=0 ; \mathrm{c}, \mathrm{d}$ ) representation of reconstructed inhomogeneity by three and one simple parametric function correspondingly.

the regularizing coefficients $\alpha_{1}, \alpha_{2}, \alpha_{3}$ (2.18). The errors of reconstruction in considered numerical example are $50 \%$ (fig. 4b) and 30\% (fig. 4c). The accuracy of reconstruction of inhomogeneities of electrical conductivity has a stronger dependence on the values of the regularizing coefficients in comparison with the elastic case. This difference can be explained by greater 


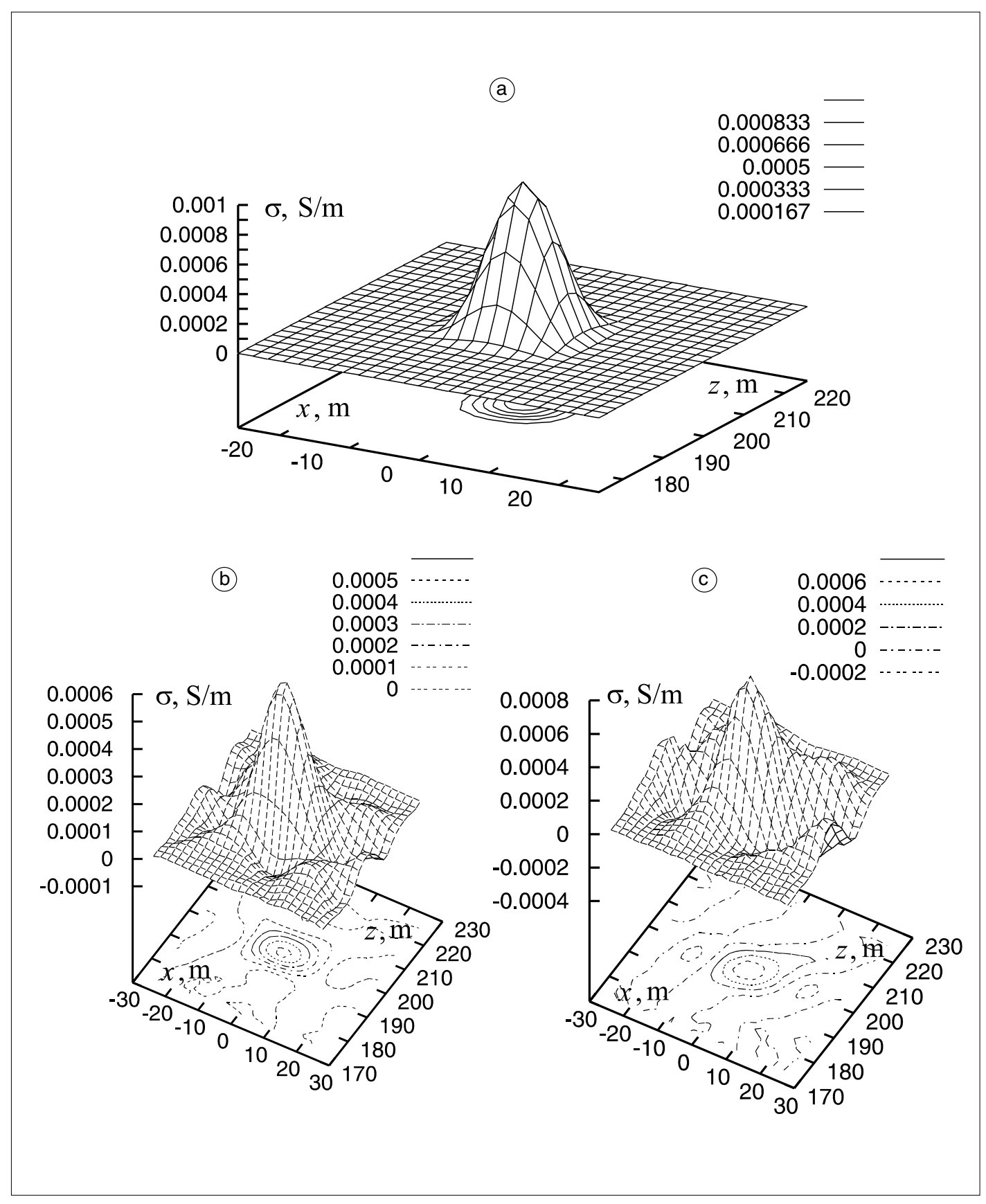

Fig. 4a-c. Reconstruction of electrical conductivity. a) The model; b,c) results of reconstruction; in the case (b) the regularizing coefficients are ten times greater than in the case (c). 
distortion of a shape of the scattered signal in the case of reconstruction of electrical conductivity.

\section{Acknowledgements}

This investigation was supported by INTAS99-1102, Russian Foundation of Basic Research (Grant 02-05-65081), Ministry of Education (Grant E00-9.0-82) and «Intergeofizika».

\section{REFERENCES}

Alumbaugh, D.L. and H.F. MorRISON (1995): Theoretical and practical consideration for crosswell electromagnetic tomography assuming a cylindrical geometry, Geophysics, 60, 846-870.

BEYLKIN, G. and R. BURRIDGE (1990): Linearized inverse scattering problems in acoustics and elasticity, Wave Motion, 12 (1), 15-52.

DEVANEY, A.J. (1984): Geophysical diffraction tomography, IEEE Trans, Geosci. Remote Sensing, GE-22 (1), 3-13.

DeVAnEY, A.J. and D. ZhANG (1991): Geophysical diffraction tomography in a layered background, Wave Motion, 14 (3), 243-265.

KELLER, J.B. (1969): Accuracy and validity of the Born and Rytov approximations, J. Opt. Soc. Am., 59, 1003-1004.

KISELEV, YU.V. and V.N. TROYAN (1997): Estimation of elastic parameters in diffraction tomography, in Proceedings of the International Seminar «Day on Diffraction-97», St. Petersburg, Russia, 3-5 June 1997, 135-139.

RYZHIKOV, G.A. and V.N. TROYAN (1994): Tomography and Inverse Sounding Problems (St. Petersburg University, St. Petersburg), pp. 220.

SAintenOY, A.C. and A. TARANTOLA (2001): Groundpenetrating radar: analysis of point diffractors for modeling and inversion, Geophysics, 66, 540-550.

SLANEY, M., A.C. KAK and L.E. LARSEN (1984): Limitation of imaging with first-order diffraction tomography, IEEE Trans. Microwave Theory Tech., MTT-32 (8), 860-874.

TroyAN, V.N. and G.A. RYZHIKov (1994): Threedimensional diffraction tomography functionals, in Proceedings of 2 nd Geophysics Congress, Thessaloniki, 340-348.

WU, R. and K. AKI (1985): Scattering characteristics of elastic waves by an elastic heterogeneity, Geophysics, 50, 585-592.

ZHOU, Q., A. BECKER and H.F. MORRISON (1993): Audiofrequency electromagnetic tomography, Geophysics, 58, 482-495. 